Brief Communication

\title{
Improved profiling of polyamines using two-dimensional gas chromatography mass spectrometry
}

\author{
Zhanru Yu' ${ }^{1}$, Honglei Huang ${ }^{1}$, Hanlin Zhang $^{2}$ and Benedikt M Kessler ${ }^{1,3}$ \\ ${ }^{1}$ TDI Mass Spectrometry Laboratory, Target Discovery Institute, Nuffield Department of \\ Medicine, University of Oxford, Oxford OX3 7FZ, UK \\ ${ }^{2}$ Kennedy Institute of Rheumatology, Nuffield Department of Orthopaedics, Rheumatology \\ and Musculoskeletal Sciences, University of Oxford, Oxford OX3 7FY
}

${ }^{3}$ Corresponding author: $\quad$ Benedikt M Kessler

Phone: +441865 612921

Email: benedikt.kessler@ndm.ox.ac.uk

Running Title: Improved polyamine profiling by GCxGC-MS

Key words: polyamines, spermidine, spermine, putrescine 2-dimensional, gas chromatography

Word counts: 2,985 


\section{ABSTRACT (153 words)}

Polyamines are a class of poly-cationic aliphatic amines, playing a role in different cellular processes such as maintaining intracellular $\mathrm{pH}$ and membrane potential that are relevant for general cellular physiology and ageing. The development of analytical methods for detection and quantitation of this class of compounds has been challenging due to the basic nature of these species. Both liquid chromatography (LC) and gas chromatography (GC) have been applied for separation, mostly coupled to mass spectrometry (MS) for detection. However, current methodologies suffer from lengthy extraction protocols and limitations in separation and detection levels. Here, we present a simplified and optimised method for straightforward extraction of polyamine metabolites including spermine, spermidine, norspermidine, cadaverine and putrescine from cellular and tissue material. We demonstrate that strong acid-based extraction and chemical derivatisation not only improves isolation, but also recovery. Combined with two-dimensional gas chromatography, this method provides clear separation and femtomole sensitivity for the profiling of polyamines. 


\section{INTRODUCTION}

Polyamines, such as spermidine, are polycationic aliphatic amines with multifunctional vital roles in general cell physiology and survival [1]. Polyamines have attracted attention for their specific involvement in stabilising DNA/RNA, regulating intracellular $\mathrm{pH}$, autophagy and aging [2]. The biosynthetic pathway involves spermidine synthase (SPDS) which catalyzes the formation of spermidine from putrescine, which is synthesized from ornithine [3]. Spermidine is a precursor to further polyamines, such as spermine and its structural isomer thermospermine. Spermidine synchronizes an array of biological processes such as the activity of $\mathrm{Ca}^{2+}, \mathrm{Na}^{+}, \mathrm{K}^{+}$-ATPases, thus maintaining membrane potential and controlling intracellular $\mathrm{pH}$ and volume. Spermine is essentially responsible for the odour of semen, but has other biological functions such as association with nucleic acids to stabilize helical structure, particularly in viruses [4]. Putrescine and cadaverine are both breakdown products of amino acids, responsible for the foul odour of putrefying flesh, but also found to be produced in the context of normal physiology, together with spermine and spermidine [5]. Due to the general role of polyamines in attenuating aging, inflammation, and its neuro- and cardioprotective effects [1], the analytical detection of polyamines has again attracted interest in recent years. Liquid chromatography (LC) $[6,7]$ and one- or two-dimensional (1D/2D) gas chromatography (GC, GCxGC) based methodologies have been developed, in most cases, coupled to mass spectrometry (MS) [8-12]. Due to the basic nature of polycations (polyamines), extraction methods out of biological material include acid-based reagents in combination with chemical derivatisation. For instance, $0.2 \%$ perfluoroheptanoic acid in methanol were shown to improve extraction efficiency by precipitating proteins, and $0.0125 \%$ perfluoroheptanoic acid in the mobile phase enhanced separation when used in combination with liquid chromatography tandem mass spectrometry (LC-MS/MS) analysis [6]. Other approaches made use of solid phase extraction (SPE) coupled online to LCMS/MS [7] or GC triple quadrupole MS [13]. Combined ion-pair extraction and GC-MS was successfully applied to the simultaneous detection of diamines, polyamines and aromatic 
amines in port wine and grape juice [14]. In this method, sample clean up consisted of the extraction of the amines with the ion-pairing reagent bis-2-ethylhexylphosphate dissolved in chloroform followed by a back-extraction with $0.1 \mathrm{M}$ hydrochloric acid $(\mathrm{HCl})$. The hydrochloric extract obtained was dried, and the amines were further derivatised with heptafluorobutyric anhydride and analysed by GC-MS in a selected ion-monitoring mode. A similar study used $\mathrm{HCl}$ extraction, ion-exchange resin catch and $\mathrm{N}$-methyl- $\mathrm{N}$-tertbutyldimethylsilyltrifluoroaceamide (MTBSTFA) derivatisation combined with GC-MS analysis [10]. Another study applied $N$-ethoxycarbonyl- $N$-pentafluoropropionyl derivatisation to detect hair polyamines by gas chromatography-mass spectrometry [9]. A recent report examined a range of acids for extraction efficiency of different polyamines [15]. However, in general, all these methods suffer from a lengthy extraction and chemical derivatisation procedure with some limitation in the sensitivity of detection.

In the present study, we describe a simplified extraction procedure based on a single-step trifluoroacetic acid (TFA) based extraction, followed by derivatisation and analysis by twodimensional (2D) GCxGC-MS. We demonstrate femtomole range detection and quantitation of a panel of polyamines isolated from tissue, cell extract and urine material.

\section{MATERIALS AND METHODS}

\section{Biological samples and chemicals}

2-Propanol and tert-butyl methyl ether were purchased from Fisher Scientific. Myristic acid$14,14,14-d_{3}$ used as an internal standard (Cambridge isotope Laboratories). Methanol, hydrochloric acid (37\%), trifluoroacetic acid (TFA) and trifluoroacetic acid anhydride (TFAA) were from Sigma-Aldrich. Polyamine standards: spermidine (SPD), spermidine- $d_{8}$, norspermidine (NSP), putrescine (PUT), cadaverine (CAD), spermine (SPM) and the nonnatural derivatives 1,7-diaminoheptane (DAH) and 1,3-dimaninopropane (DAP) were also purchased from Sigma Aldrich. A stock solution was prepared at $0.1 \mathrm{M}$ final concentration in 
$0.5 \mathrm{M} \mathrm{HCl}$ for each polyamine standard. Four standard solutions $(0.01,0.1,1,10 \mathrm{mM})$ were made and used for the quantitation measurements by dilution of the stock solutions. Jurkat $T$ lymphoblastoid cell line (ATCC TIB-152) was cultured in RPMI 1640 medium (Gibco)

supplemented with $10 \%$ fetal calf serum (Sigma-Aldrich), $1 \%$ glutamine and $1 \%$ penicillin/streptomycin (Sigma-Aldrich). C57/B6 mice were held at the Wellcome Centre for Human Genetics, (Oxford University), and the animal material used in this study was obtained in accordance with Home Office guidance on operation of animals (ethics number PPL 30/2750).

\section{Polyamine extraction protocol}

For brain tissue or cell samples, $\sim 50 \mathrm{mg}$ of wet brain tissue or 5 million Jurkat cells were used for the extraction. Cells were collected by centrifugation for five minutes at $1,500 \mathrm{~g}$, followed by one round of washing using PBS. Brain tissue or cell pellet was re-suspended in $800 \mu \mathrm{L}$ of lysis buffer (5\% TFA, $15 \% \mathrm{H}_{2} \mathrm{O}$ and $80 \%$ methanol) spiked with $5 \mu \mathrm{L}$ of DAH or spermidine- $d_{8}$ in methanol $(1 \mathrm{mg} / \mathrm{mL})$. In the recovery experiments, these samples were spiked with SPD, SPM, DAP, DAH, CAD, NSP and PUT (10 $\mu \mathrm{L}$ of each polyamine standard

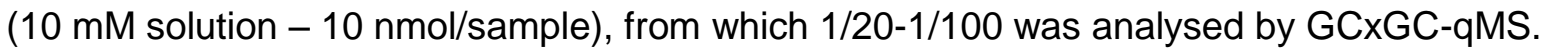
The suspensions were homogenised in a bead beater (Precellys 24, Bertin Technologies) for four cycles $(6500 \mathrm{~Hz}, 45 \mathrm{~s})$ with one minute of dry ice incubation between cycles. After kept at $-80{ }^{\circ} \mathrm{C}$ for one hour, the samples were centrifuged at $13,000 \mathrm{~g}$ for 20 minutes at $4{ }^{\circ} \mathrm{C}$. The supernatant was collected and dried overnight. The dried samples were stored at $-80{ }^{\circ} \mathrm{C}$ until use. In case of pig urine samples, $150 \mu \mathrm{L}$ of biofluid was re-suspended in $850 \mu \mathrm{L}$ of lysis buffer (5\%TFA, $15 \% \mathrm{H}_{2} \mathrm{O}, 80 \%$ methanol) spiked with $5 \mu$ of DAH or spermidine- $\mathrm{d}_{8}$ in methanol $(1 \mathrm{mg} / \mathrm{mL})$. In the recovery experiments, $10 \mu \mathrm{L}$ of each polyamine standard (10 $\mathrm{mM}-10 \mathrm{nmol}$ / sample) was spiked into the samples, from which 1/20-1/100 was analysed by GCxGC-qMS. The extraction was performed as described for brain tissue and cell samples. 


\section{Derivatisation protocol}

Chemical derivatisation was performed in a simplified version as to a protocol reported previously [16]. In brief, samples were resuspended in $100 \mu \mathrm{L}$ of ethyl acetate and $200 \mu \mathrm{L}$ TFAA. The tubes were sealed and incubated for $1 \mathrm{~h}$ at $60^{\circ} \mathrm{C}$. The mixture was subsequently evaporated to dryness by vacuum centrifugation. Derivatised samples were then resuspended in $30 \mu \mathrm{L}$ of 2 -propanol per $100 \mu \mathrm{L}$ urine, $5^{\star} 10^{6}$ cells or $30 \mathrm{mg}$ tissue and subsequently analysed by GCxGC-qMS.

\section{Analysis by GCxGC-qMS and data analysis}

Samples were directly injected into a Shimadzu 20i/s auto sampler / two-dimensional gas chromatograph system coupled to a single quadrupole mass spectrometer GCxGC-qMS (Shimadzu QP2010 Ultra) as described previously [17]. Raw GCxGC-MS data were analysed using GCMSsolution software (v2.72/4.20) and Chromsquare software (v2.1.6, Shimadzu) and GC Image (v2.3), and metabolite identification was performed using the NIST 11/s, OA_T, FA_ME and YUTIDI in-house libraries. Annotation of metabolites was carried out by comparing electron impact (EI) spectra and retention times to polyamine standards.

\section{RESULTS \& DISCUSSION}

Traditional protocols established for metabolite analysis based on the extraction of the mixture of methanol and water followed by trimethylsilylation are suboptimal for the detection of polyamines, including spermidine and spermine, due to their charged state at physiological $\mathrm{pH}$ that renders them less soluble in organic solvent $[16,17]$. With the aim to establish a simple, more effective extraction protocol applicable to biological material, we reasoned that a strong acid such as TFA in water / methanol would be applicable in a 
simplified experimental workflow combined with a trifluoroacetic anhydride (TFAA)-based derivatisation approach as shown in Figure 1A. TFAA-based derivatives are more stable than silylated functional groups. To test this and to ensure appropriate identification, we spiked a human Jurkat cell line extract with a mixture of standards including spermidine (SPD), spermine (SPM), putrescine (PUT), cadaverine (CAD), norspermidine (NSP), 1,3dimaninopropane (DAP) (exists in plants and microorganisms, useful reference compound in the context of plant extractions [18]) and the non-natural derivative 1,7-diaminoheptane (DAH) as an internal synthetic standard. We found that polyamine standards can be clearly separated and identified by one- and two-dimensional gas chromatography coupled to single quadrupole mass spectrometry (GCxGC-qMS) (Figures 1B, S1A). We were able to define diagnostic fragment ion panels unique to each polyamine for single ion monitoring (SIM) based detection and quantitation (Table S1) based on electron impact mass spectrometry (EI-MS) spectra of sufficient quality for the characterisation of and distinction between individual polyamines (Figures S1B-H). The amount of TFA in the water/methanol extraction buffer $(1.25 \%-10 \%)$ did not affect extraction efficiency to a great extent, although we observed yellow precipitate material at the lowest TFA levels (Figure S2A). 5\% TFA was considered optimal, and we further compared the efficacy of polyamine extraction between increasing proportions of methanol present in the extraction buffer. We found that using $80 \%$ methanol yielded in the best extraction efficiency of all polyamines (Figure S2B). To extend this, we examined polyamine metabolite recovery from complex biological matrices such as mouse brain and Jurkat human T-cell extracts. A recovery test in which we spiked the extract material with known amounts of the polyamines diaminopropate (DAP), putrescine (PUT), cadaverine (CAD), diaminoheptane (DAH), norspermidine (NSP), spermidine (SPD) and spermine (SPM) revealed recovery yields between $87 \%$ - 99\% when compared to direct injection of standards after TFA derivatisation (Figure 2A). To further examine efficiency and to compare the extent of improvement over a conventional extraction protocol, we performed two subsequent extraction steps to measure left over material after each round of extraction. When an extraction buffer containing $5 \%$ TFA, $15 \% \mathrm{H}_{2} \mathrm{O}$ and $80 \%$ methanol was used as the 
first step, a nearly complete recovery of all polyamine material was achieved, tested here with putrescine, spermidine and spermine spiked into Jurkat T cell extracts. In contrast, when a conventional extraction buffer ( $80 \%$ methanol in water) was used as the first step, followed by a TFA containing buffer, recovery rates were markedly inferior (Figure 2B). Finally, the first extraction step yielded in $>99 \%$ recovery of spermidine and spermine as subsequent extractions did not result in the isolation of remaining material (Figure S2C). Taken together, we conclude to have optimised a single-step extraction protocol for the robust and quantitative isolation of polyamines from a variety of biological materials. We next explored the sensitivity and dynamic range of linear quantitation that can be achieved with our method when analysed with GCxGC-qMS. To this end, standard dilution series were prepared and analysed by GCxGC-qMS. We detected all polyamines with a limit of detection (L.O.D.) in the range of $140 \mathrm{fmol}$ (SPD) and $670 \mathrm{fmol}$ (DAP) which were calculated according to previous studies $[17,19,20]$ (Figure S3A-G). The linear dynamic range was greatest for $\operatorname{SPD}\left(2^{*} 10^{3}\right)$ and between $1 * 10^{2}$ and $1 * 10^{3}$ for all other polyamines. Having established this protocol, we applied our method to profile the natural abundance of polyamines in human and animal sample specimens. 2D-GCxGC-qMS analysis detected putrescine, spermine and spermidine (DAH internal standard) in human Jurkat T-cells that can be clearly distinguished as single blobs, indicating that although the polyamines showed distinct retention times using one dimension, the second dimension GCxGC further separated them from other endogenous metabolite species (Figure 3A). In addition, analysis of brain extracts obtained from C57/B6 mice and pig urine material also revealed detection of endogenous levels of polyamines such as putrescine, spermidine and spermine (Figure 3B). The natural abundance levels of putrescine PUT, spermidine SPD and spermine SPM were calculated to be in the range of $32.5-1200.6 \mathrm{pmol} / 10^{6}$ cells and $12.6-159.2 \mathrm{pmol} / \mathrm{mg}$ wet brain tissue (Table 1) in agreement with previous studies [21-23], whereas it was borderline for detection in pig urine (Figure 3B). In summary, this method is simple, robust and orthogonal to classical extraction protocols used in GC- based metabolomics studies. We have demonstrated its usefulness for the 
analysis of cellular and tissue extracts as well as urine material, thereby expanding the metabolic coverage achievable by this analytical platform.

\section{ACKNOWLEDGEMENTS}

We thank members of the Kessler group and Anna Katharina Simon for helpful discussions.

This work was supported by the Wellcome (097813/Z/11/Z) and the Engineering and Physical Sciences Research Council (EP/N034295/1) to B.M.K.

\section{REFERENCES}

[1] F. Madeo, T. Eisenberg, F. Pietrocola, G. Kroemer, Spermidine in health and disease, Science (80-. ). 359 (2018). doi:10.1126/science.aan2788.

[2] R.A. Casero, L.J. Marton, Targeting polyamine metabolism and function in cancer and other hyperproliferative diseases, Nat. Rev. Drug Discov. 6 (2007) 373-390. doi:10.1038/nrd2243.

[3] D. Ramani, J.P. De Bandt, L. Cynober, Aliphatic polyamines in physiology and diseases, Clin. Nutr. 33 (2014) 14-22. doi:10.1016/j.clnu.2013.09.019.

[4] T. Ikemura, The relation between the strong binding of spermine to polynucleotides and the conformation of polynucleotides, BBA Sect. Nucleic Acids Protein Synth. 195 (1969) 389-395. doi:10.1016/0005-2787(69)90645-5.

[5] H.A. Urlings, N.G. Fransen, P.G. Bijker, J.G. van Logtestijn, Proteolysis and amino acid breakdown of heated and irradiated poultry byproducts and muscle tissue., J. Anim. Sci. 71 (1993) 2432-2438. 
[6] C. Yu, R. Liu, C. Xie, Q. Zhang, Y. Yin, K. Bi, Q. Li, Quantification of free polyamines and their metabolites in biofluids and liver tissue by UHPLC-MS/MS: application to identify the potential biomarkers of hepatocellular carcinoma, Anal. Bioanal. Chem. 407 (2015) 6891-6897. doi:10.1007/s00216-015-8860-6.

[7] C. Magnes, A. Fauland, E. Gander, S. Narath, M. Ratzer, T. Eisenberg, F. Madeo, T. Pieber, F. Sinner, Polyamines in biological samples: Rapid and robust quantification by solid-phase extraction online-coupled to liquid chromatography-tandem mass spectrometry, J. Chromatogr. A. 1331 (2014) 44-51. doi:10.1016/j.chroma.2013.12.061.

[8] M.J. Paik, S. Lee, K.H. Cho, K.R. Kim, Urinary polyamines and N-acetylated polyamines in four patients with Alzheimer's disease as their $\mathrm{N}$-ethoxycarbonyl-Npentafluoropropionyl derivatives by gas chromatography-mass spectrometry in selected ion monitoring mode, Anal. Chim. Acta. 576 (2006) 55-60. doi:10.1016/j.aca.2006.01.070.

[9] M.H. Choi, K.R. Kim, B.C. Chung, Determination of hair polyamines as Nethoxycarbonyl-N-pentafluoropropionyl derivatives by gas chromatography-mass spectrometry, J. Chromatogr. A. 897 (2000) 295-305. doi:10.1016/S00219673(00)00800-1.

[10] P.L. Wood, M.A. Khan, J.R. Moskal, Neurochemical analysis of amino acids, polyamines and carboxylic acids: GC-MS quantitation of tBDMS derivatives using ammonia positive chemical ionization, J. Chromatogr. B Anal. Technol. Biomed. Life Sci. 831 (2006) 313-319. doi:10.1016/j.jchromb.2005.12.031.

[11] S. Stadler, P.H. Stefanuto, J.D. Byer, M. Brokl, S. Forbes, J.F. Focant, Analysis of synthetic canine training aids by comprehensive two-dimensional gas chromatography-time of flight mass spectrometry, J. Chromatogr. A. (2012). doi:10.1016/j.chroma.2012.04.001.

[12] L. Li, K. Hara, J. Liu, Y. Yu, L. Gao, Y. Wang, Y. Wang, Rapid and simultaneous determination of hair polyamines as $\mathrm{N}$-heptafluorobutyryl derivatives by gas 
chromatography-mass spectrometry, J. Chromatogr. B Anal. Technol. Biomed. Life Sci. 876 (2008) 257-260. doi:10.1016/j.jchromb.2008.10.054.

[13] A. Naccarato, R. Elliani, B. Cavaliere, G. Sindona, A. Tagarelli, Development of a fast and simple gas chromatographic protocol based on the combined use of alkyl chloroformate and solid phase microextraction for the assay of polyamines in human urine, J. Chromatogr. A. 1549 (2018) 1-13. doi:https://doi.org/10.1016/j.chroma.2018.03.034.

[14] J.O. Fernandes, M. a Ferreira, Combined ion-pair extraction and gas chromatography, mass spectrometry for the simultaneous determination of diamines, polyamines and aromatic amines in Port wine and grape juice, J. Chromatogr. A. 886 (2000) 183-195. doi:http://dx.doi.org/10.1016/S0021-9673(00)00447-7.

[15] F.B. Custódio, É. Tavares, M.B.A. Glória, Extraction of bioactive amines from grated Parmesan cheese using acid, alkaline and organic solvents, J. Food Compos. Anal. 20 (2007) 280-288. doi:10.1016/j.jfca.2006.06.009.

[16] G.G. Chen, G. Turecki, O.A. Mamer, A quantitative GC-MS method for three major polyamines in postmortem brain cortex, J. Mass Spectrom. 44 (2009) 1203-1210. doi:10.1002/jms.1597.

[17] Z. Yu, H. Huang, A. Reim, P.D. Charles, A. Northage, D. Jackson, I. Parry, B.M. Kessler, Optimizing 2D gas chromatography mass spectrometry for robust tissue, serum and urine metabolite profiling, Talanta. 165 (2017) 685-691. doi:10.1016/j.talanta.2017.01.003.

[18] R.D. Slocum, R. Kaur-Sawhney, A.W. Galston, The physiology and biochemistry of polyamines in plants, Arch. Biochem. Biophys. (1984). doi:10.1016/00039861(84)90201-7.

[19] A. Shrivastava, V. Gupta, Methods for the determination of limit of detection and limit of quantitation of the analytical methods, Chronicles Young Sci. (2011). doi:10.4103/2229-5186.79345.

[20] A.P. Siskos, P. Jain, W. Römisch-Margl, M. Bennett, D. Achaintre, Y. Asad, L. 
Marney, L. Richardson, A. Koulman, J.L. Griffin, F. Raynaud, A. Scalbert, J. Adamski,

C. Prehn, H.C. Keun, Interlaboratory Reproducibility of a Targeted Metabolomics Platform for Analysis of Human Serum and Plasma, Anal. Chem. (2017). doi:10.1021/acs.analchem.6b02930.

[21] H. Antrup, N. Seiler, On the turnover of polyamines spermidine and spermine in mouse brain and other organs, Neurochem. Res. 5 (1980) 123-143. doi:10.1007/BF00964327.

[22] R.J. Bergeron, W.R. Weimar, R. M??ller, C.O. Zimmerman, B.H. McCosar, H. Yao, R.E. Smith, Effect of polyamine analogues on hypusine content in JURKAT T-cells, J. Med. Chem. 41 (1998) 3901-3908. doi:10.1021/jm980390o.

[23] Y. Ikeguchi, X. Wang, D.E. McCloskey, C.S. Coleman, P. Nelson, G. Hu, L.M. Shantz, A.E. Pegg, Characterization of transgenic mice with widespread overexpression of spermine synthase., Biochem. J. 381 (2004) 701-707. doi:10.1042/BJ20040419.

\section{FIGURE LEGENDS}

\section{Figure 1. Simple and robust workflow for profiling polyamines via GCXGC-qMS.}

(A) Schematic representation of the experimental procedure described in this study. (B) A representative one-dimensional gas chromatography coupled to single quadrupole mass spectrometry (GCxGC-qMS) analysis of polyamine standards spiked into a Jurkat cell extract. The blobs characterised as 1,3-diaminopropane-2TFA (DAP-2TFA), putrescine2TFA (PUT-2TFA), cadaverine-2TFA (CAD-2TFA), 1,7-dimaninoheptane-2TFA (DAH2TFA), norspermidine-2TFA (NSP-3TFA), spermidine-3TFA (SPD-3TFA) and spermine4TFA (SPM-4TFA) are labelled with rectangles (see Figure S1A-G for the El spectra used for identification). Insert: 2-dimensional GCxGC-qMS analysis; colours reflect peak intensities. 
Figure 2. Single-step complete polyamine recovery from cellular \& brain extracts and pig urine.

(A) Recovery of polyamines DAP, PUT, CAD, DAH, NSP, SPD and SPM spiked into extracts prepared from mice brain and Jurkat T cells. TFA/methanol extractions were performed using $5 \%$ TFA, $15 \% \mathrm{H}_{2} \mathrm{O}$ and $80 \%$ methanol, followed by chemical derivatisation using TFAA and analysis by GCxGC-qMS. MS signal intensities were compared to metabolite standards alone to calculate \% recovery. (B) Recovery test using two consecutive extraction steps with a conventional ( $80 \%$ methanol in water - method A) and the new extraction buffer (5\% TFA, $15 \% \mathrm{H}_{2} \mathrm{O}$ and $80 \%$ methanol - method $\mathrm{B}$ ).

Figure 3. Profiling endogenous polyamines in Jurkat cell lysates, brain extracts and pig urine.

(A) TFA/methanol extraction was performed on Jurkat T cell extracts, followed by TFAA chemical derivatisation and analysis by GCxGC-qMS. A representative 2D-GCxGC chromatogram is shown, and the identified TFA derivatised polyamines putrescine, spermidine and spermine are indicated (diaminoheptane used as internal standard). (B) 1DSIM (single ion monitoring) chromatograms are shown for the detection of endogenous spermine (top panel), spermidine (middle panel) and putrescine (lower panel). Endogenous polyamines were detected in human Jurkat T-cell extracts (black), C57/B6 mouse brain extracts (red) and in trace amounts in pig urine (blue).

Table 1. Detection of endogenous polyamines by GCxGC-qMS. 
Table 1: Detection of endogenous polyamines by GCxGC-qMS

\begin{tabular}{llll} 
Polyamines & Putrescine & Spermidine & Spermine \\
$\begin{array}{l}\text { Jurkat Cell Extracts } \\
\text { (pmol/106 cells) }\end{array}$ & $32.5 \pm 2.6$ & $859.6 \pm 71.4$ & $1200.6 \pm 125.8$ \\
$\begin{array}{l}\text { Mouse Brain Extracts } \\
\text { (pmol/mg wet tissue) }\end{array}$ & $12.6 \pm 1.3$ & $215.1 \pm 16.2$ & $159.2 \pm 10.7$ \\
\hline
\end{tabular}


Figure 1

A

Homogenization of cells in TFA (5\%) by bead beater
Phase separation by centrifugation
Collect

supernatant and dry it under vacuum

\section{Data Analysis by GCM solution,}

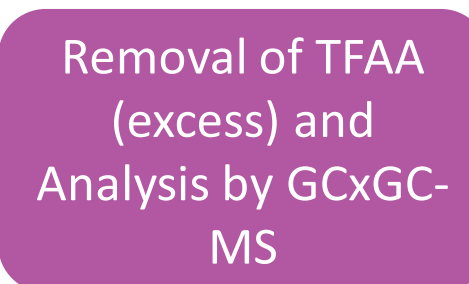

Derivatise with TFAA into TFA Amide
B

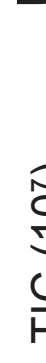

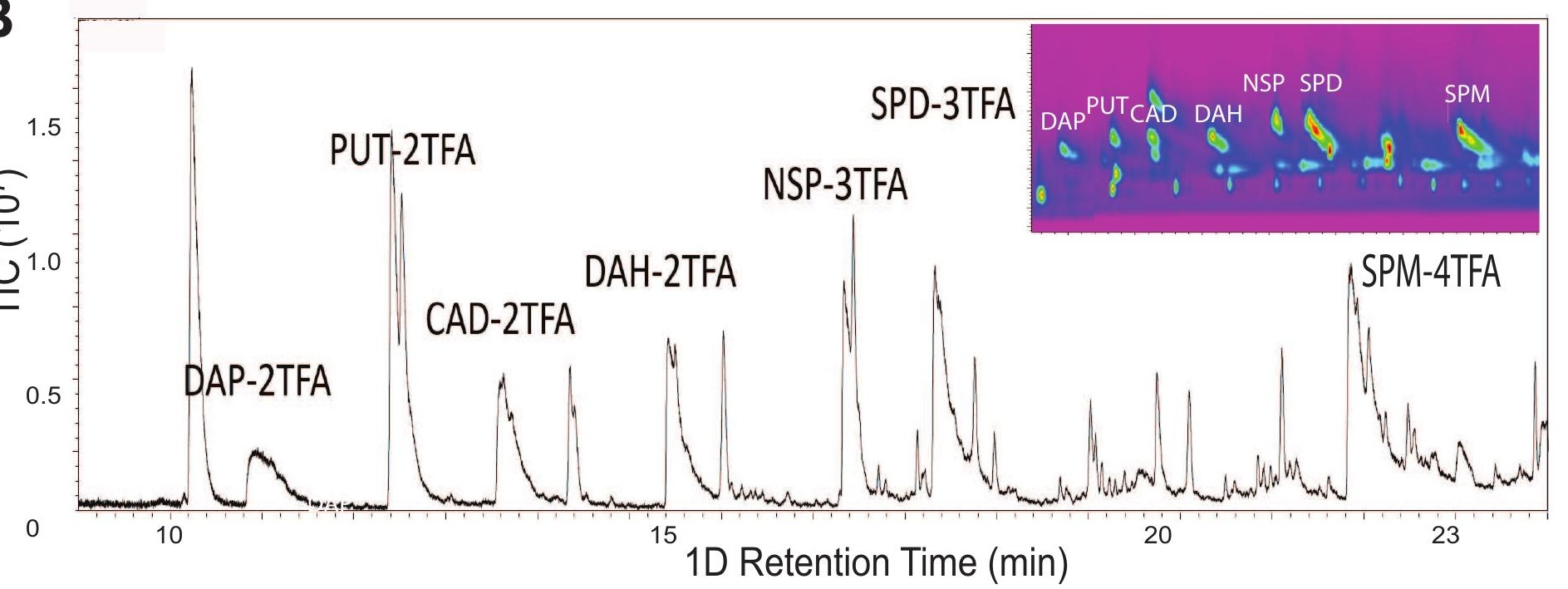


Figure 2

Figure 2
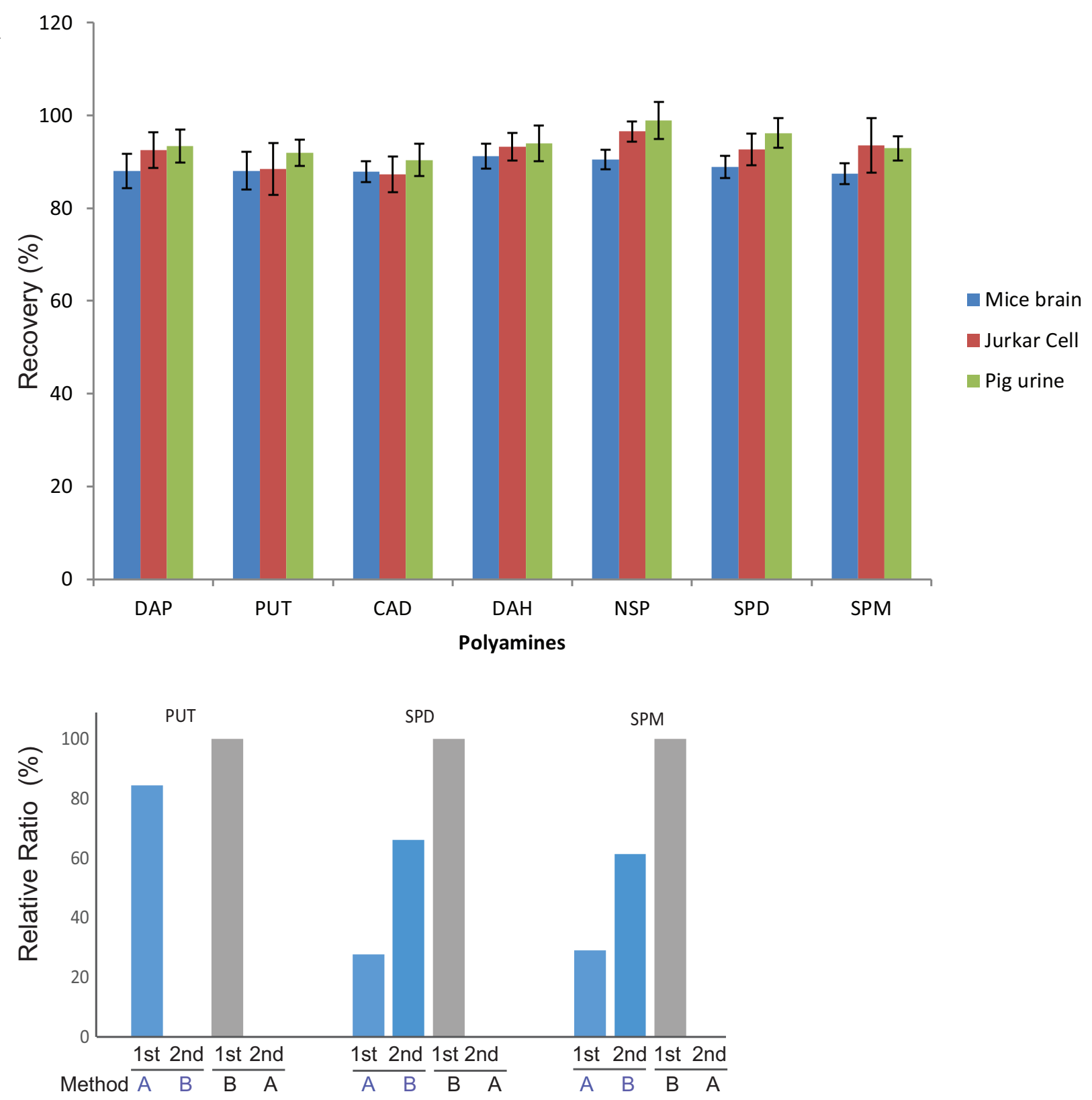
Checklist

Click here to download Supplementary Material: Checklist_REVISED.docx 


\section{List of Three Potential Reviewers}

\section{Professor Luigi Mondello}

Dipartimento di Scienze Chimiche

University of Messina

Italy

Email:Imondello@unime.it

Reason: Expert in the field of GCxGC-MS

Professor Maria I Klapa

Metabolic Engineering and Systems Biology Laboratory

Institute of Chemical Engineering Sciences

Patras, Greece

Email:mklapa@iceht.forth.gr

Reason: Expert in biological metabolomics and GC-MS technology

Professor Daniel Raftery

Department of Chemistry

Purdue University, Matrix-Bio, Inc, West Lafayette, Indiana 47907

USA

Email: raftery@purdue.edu

Reason: Expert in GCXGC-MS and medical metabolomics 
STATEMENT OF COMPETING INTERESTS
The authors declare no competing interests related to the work described in this manuscript.

STATEMENT OF COMPETING INTERESTS
The authors declare no competing interests related to the work described in this manuscript.

STATEMENT OF COMPETING INTERESTS
The authors declare no competing interests related to the work described in this manuscript.

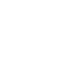

\section{Author Agreement}

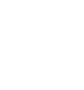

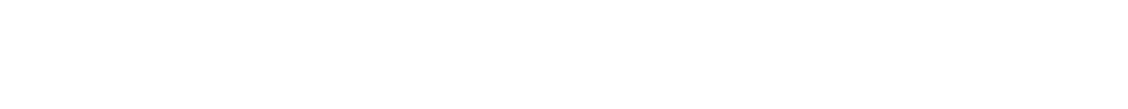

.

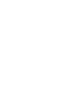

(2)

.

(1)

(1)

(1)
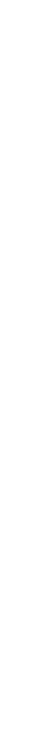
Credit Author Statement

\section{AUTHOR CONTRIBUTIONS}

ZY and BMK conceptualised the study. ZY, HZ and HH performed experiments. $\mathrm{ZY}, \mathrm{HH}$ and BMK analysed the mass spectrometry data. BMK and ZY wrote the manuscript, and all authors made comments and suggestions. 
Supplementary Material (figures)
Click here to download Supplementary Material: All_Supplementary_Figs_Yu_REVISED_FINAL.pdf

(a)

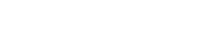

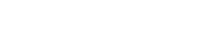

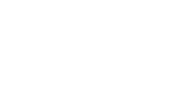

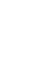


Supplementary Material (table)
Click here to download Supplen

Click here to download Supplementary Material: Table S1.pdf

\section{df}

(2) 